DFTT-40/93

hep-th/9307170

July 1993

\title{
Classification of Structure Constants for W-algebras from Highest Weights
}

\author{
K. Hornfeck \\ INFN, Sezione di Torino, Via Pietro Giuria 1, I-10125 Torino, Italy
}

\begin{abstract}
We show that the structure constants of W-algebras can be grouped according to the lowest (bosonic) spin(s) of the algebra. The structure constants in each group are described by a unique formula, depending on a functional parameter $h(c)$ that is characteristic for each algebra. As examples we give the structure constants $\mathcal{C}_{33}^{4}$ and $\mathcal{C}_{44}^{4}$ for the algebras of type $\mathrm{W}(2,3,4, \ldots)$ (that include the $\mathrm{WA}_{n-1}$-algebras) and the structure constant $\mathcal{C}_{44}^{4}$ for the algebras of type $\mathrm{W}(2,4, \ldots)$, especially for all the algebras $\mathrm{WD}_{n}, \mathrm{WB}(0, n), \mathrm{WB}_{n}$ and $\mathrm{WC}_{n}$. It also includes the bosonic projection of the super-Virasoro algebra and a yet unexplained algebra of type $\mathrm{W}(2,4,6)$ found previously.
\end{abstract}

\footnotetext{
*e-mail: HORNFECK@TO.INFN.IT; 31890::HORNFECK
} 


\section{Introduction}

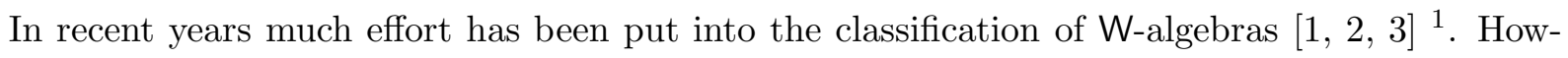
ever, there seem still to be cases that withstand such classifications. One example occurs for W-algebras of type $\mathrm{W}(2,4,6)$ that show four solutions [5]: Two have been identified with the algebras $\mathrm{WB}_{3}$ and $\mathrm{WC}_{3}$ and a third solution is the bosonic projection of the super-Virasoro algebra [6, []. But a fourth algebra exists that has not yet found an explanation [8]. So far the only way to decide definitely the number of solutions of a general $\mathrm{W}$-algebra of type $\mathrm{W}\left(2, d_{1}, d_{2}, \ldots\right)$ is by explicit construction, a task that would go soon beyond the available computer power.

In this paper we shall start from a different point of view that describes the known $\mathrm{W}$-algebras based on the simple Lie-algebras but also allows for additional solutions. Especially it includes the fourth solution of $\mathrm{W}(2,4,6)$ in a very surprising (though at the moment only formal) way.

Computing structure constants by solving Jacobi-identites in the usual way always makes use of the whole set of fields of the algebra. As a consequence, even structure constants involving only low-dimensional fields are impossible to obtain in the conventional way if the algebra contains many or "high"-dimensional fields. In this way already the algebra of type $\mathrm{W}(2,4,6)$ is almost at the limit that can be accessed with standard methods.

Contrary to that, our approach will only involve Jacobi-identities with those fields that enter in the structure constants in which one is interested. Starting from the highest weights [9] for the W-algebra we find that a set of highest weights exists that considerably simplify the construction of the structure constants of the algebra.

In section 2 we explain our philosophy in the case of the $\mathrm{W}_{3}$-algebra and in the following sections 3 to 5 we apply our ideas to more complicated $\mathrm{W}$-algebras and give some examples for structure constants that will include the simplest ones for the algebras $\mathrm{WA}_{n-1}, \mathrm{WB}_{n}, \mathrm{WC}_{n}, \mathrm{WD}_{n}$ and $\mathrm{WB}(0, n)$ as functions of $n$.

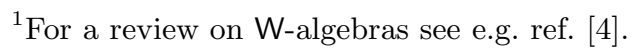




\section{Preliminaries: The $\mathrm{W}_{3}$-algebra}

The $\mathrm{W}_{3}$-algebra of Zamolodchikov [10] is certainly one of the best investigated $\mathrm{W}$-algebras. Because of its relative simplicity we shall start our discussion with it.

A highest weight state $|h, w\rangle$ is a primary state, i.e.

$$
\begin{array}{ll}
L_{n} \mid h, w>=0 & \text { for } n>0 \\
L_{o}|h, w>=h| h, w> &
\end{array}
$$

and is an eigenstate of $W_{o}$, whereas it is annihilated by the positive frequency operators $W_{n}$ :

$$
\begin{array}{ll}
W_{n} \mid h, w>=0 & \text { for } n>0 \\
W_{o}|h, w>=w| h, w>. &
\end{array}
$$

In [11] the degenerate representations have been investigated. By applying the operators $L_{n}$ and $W_{n}$ (with $\left.n<0\right)$ to the highest weight states $\mid h, w>$ we can produce other primary states. At the first level it is of the form

$$
\chi_{1}=\left(2 h W_{-1}-3 w L_{-1}\right) \mid h, w>
$$

and at the second level

$$
\chi_{2}=\left(h(5 h+1) W_{-2}-12 w L_{-1}^{2}+6 w(h+1) L_{-2}\right) \mid h, w>.
$$

For specific values of $h$ and $w$ these states become null-states and the representation is degenerate. The state $\chi_{1}$ is a null-state whenever

$$
w^{2}=-\frac{2 h^{2}(c-32 h-2)}{9(5 c+22)}
$$

and $\chi_{2}$ is a null-state if $w$ is given by eq. (2.5) and the conformal dimension $h$ of the highest weight state obeys the quadratic equation

$$
0=\left(h+1-\frac{16}{3} \alpha_{+}^{2}\right)\left(h+1-\frac{16}{3} \alpha_{-}^{2}\right)=h^{2}+\frac{h}{18}(c-14)+\frac{1}{18} c .
$$


It follows that whenever $\chi_{2}$ is a null-state, also $\chi_{1}$ is automatically a null-state (and the representation is completely degenerate).

The state $|h, w\rangle$ can be created by a field $P_{o}(z)$,

$$
\left|h, w>=\lim _{z \rightarrow 0} P_{o}(z)\right| 0>
$$

and the highest-weight conditions (2.1) and (2.2) translate into the condition that $P_{o}$ is a primary field of dimension $h$ and in the operator product expansion (OPE) of $W$ with $P_{o}$ appears as lowest-dimensional field $P_{o}$ itself with eigenvalue $w$, but also higher-dimensional fields can occur,

$$
\begin{aligned}
T \star P_{o} & =h P_{o} \\
W \star P_{o} & =w P_{o}+P_{1}+P_{2},
\end{aligned}
$$

where $P_{1}$ and $P_{2}$ are new primary fields of dimension $h+1$ and $h+2$, respectively. They are no longer highest weights, since in the OPE of $W$ with $P_{1}$ and $P_{2}$ also $P_{o}$ will in general appear. These new fields can be in some sense regarded to be connected to the states $\chi_{1}$ and $\chi_{2}$. We expect therefore that in the case where $\chi_{1}$ and $\chi_{2}$ are null-fields, that is eqs. (2.5) and (2.6) are satisfied, the OPEs (2.8) reduce to 2

$$
\begin{aligned}
T \star P_{o} & =h P_{o} \\
W \star P_{o} & =w P_{o} .
\end{aligned}
$$

We can also make the ansatz (2.9) to our starting point and ask when it is consistent by evaluating the Jacobi-identity $\left(W, W, P_{o}\right)$. This Jacobi-identity then reproduces exactly eqs. (2.5) and (2.6) 3 .

\footnotetext{
${ }^{2}$ However, using the free-field realization for the $W_{3}$-algebra and the vertex operator $: \exp i \vec{\beta} \vec{\phi}:$ for $P_{o}$ (for details see 11), one can see that this statement is not completely true. Even when $\chi_{1}$ and $\chi_{2}$ are null-states, non-zero primary fields of dimension $h+1$ and $h+2$ can be constructed. But the free-field realization also justifies eq. 2.9.). On the other hand, in principle the null-state conditions are not necessary conditions for the OPEs 2.9).

${ }^{3}$ Remember that one Jacobi-identity produces in general a set of constraints.
} 


\section{Adding more fields to $\mathrm{W}_{3}$}

We want to consider now a more general $\mathrm{W}$-algebra that has a spin-3 field $W_{3}$, a spin-4 field $W_{4}$ and an arbitrary set of other primary fields with dimension $>4$. We shall denote this algebra as $\mathrm{W}(2,3,4, \ldots)$ and examples for such an algebra are the $\mathrm{WA}_{n-1}$-algebras. The general OPEs of the low-dimensional fields are

$$
\begin{aligned}
W_{3} \star W_{3} & =\frac{c}{3} I+\sqrt{\mathcal{A}} W_{4} \\
W_{3} \star W_{4} & =\frac{3}{4} \sqrt{\mathcal{A}} W_{3}+W_{5}+W_{6,1}
\end{aligned}
$$

The structure constant $\mathcal{A}$ depends on the actual algebra; we have not assigned structure constants to $W_{5}$ and $W_{6,1}$, because in our discussion will not enter their normalisation-even not their existence or the number of spin- 5 and spin- 6 fields.

We are again interested in the case of the complete degenerate representation, when the lowestlevel states become null-states. Instead of the ansatz (2.9) we have now

$$
\begin{aligned}
& W_{3} \star P_{o}=w_{3} P_{o} \\
& W_{4} \star P_{o}=w_{4} P_{o}+q_{4} P_{3} \\
& W_{5} \star P_{o}=w_{5} P_{o}+\ldots .
\end{aligned}
$$

Here $P_{3}$ is no longer a new field (as were $P_{1}$ and $P_{2}$ in the example with the $\mathrm{W}_{3}$-algebra) but the composite primary field

$$
P_{3}=: W_{3} P_{o}:+w_{3} s_{1}(h, c): T^{\prime} P_{o}:+w_{3} s_{2}(h, c): T P_{o}^{\prime}:+w_{3} s_{3}(h, c) P_{o}^{\prime \prime \prime}
$$

that cannot be neglected from the OPE (3.2). Similary, the "..." in eqs. (3.2) stand for other composite fields of the same kind that do not enter the calculation up to the point we want to carry out here, but would be needed for consistency. The Jacobi-identity $\left(W_{3}, W_{3}, P_{o}\right)$ already fixes the eigenvalues $w_{3}$ and $w_{4}$ for any of these algebras,

$$
\begin{aligned}
& w_{3}^{2}=\frac{2 h^{2}[c(1+2 h)+2 h(8 h-5)]}{9[c(3-2 h)+2 h]} \\
& w_{4}=\frac{8(2+c) h[c(1+h)+2 h(9 h-7)]}{\sqrt{\mathcal{A}}(22+5 c)[c(3-2 h)+2 h]},
\end{aligned}
$$


and the Jacobi-identity $\left(W_{3}, W_{4}, P_{o}\right)$ yields $W_{6,1}=0$ and

$$
\begin{aligned}
w_{5} & =\frac{48(2+c)[c(1+h)+9 h(2 h-7)][c(3+2 h)+2 h(32 h-27)] w_{3}}{\sqrt{A}(114+7 c)[c(3-2 h)+2 h][c(2 h+1)+2 h(8 h-5)]} \\
q_{4} & =\frac{144(h-1) w_{3}}{\sqrt{\mathcal{A}}[c(2 h+1)+2 h(8 h-5)]}
\end{aligned}
$$

and a third order equation for $h(\mathcal{A}, c)$.

On the other hand, $h(\mathcal{A}, c)$ can be inverted to give the structure constant $\mathcal{A}$ as a function of the dimension $h$ of the highest weight satisfying eq. (3.2),

$$
\mathcal{A}(h)=\frac{256(2+c)[c(h-2)-2 h][c(h+1)+2 h(9 h-7)]}{(22+5 c)[c(2 h-3)-2 h][c(2 h+1)+2 h(8 h-5)]}
$$

Including also the OPE

$$
W_{4} \star W_{4}=\frac{c}{4} I+\mathcal{B} \sqrt{\mathcal{A}} W_{4}+W_{6,2}
$$

we also find the form of the structure constant $\mathcal{B}$ from the Jacobi-identity $\left(W_{4}, W_{4}, P_{o}\right)$ :

$\mathcal{B}(h)=\frac{3\left[c^{3}\left(2 h^{2}-2 h-9\right)+2 c^{2}\left(38 h^{3}-131 h^{2}+75 h-3\right)-4 c h\left(45 h^{2}+17 h-47\right)-8 h^{2}(41 h-38)\right]}{8(2+c)[c(h-2)-2 h][c(h+1)+2 h(9 h-7)]}$

The highest weight $h$ entering in these equations will be in general a function of the central charge $c$. Contrary to the $\mathrm{W}_{3}$-algebra (and later to the algebras of type $\mathrm{W}(2,5, \ldots)$ and type $\mathrm{W}(2,6, \ldots))$ it is not fixed by the Jacobi-identities and one could imagine that with an arbitrary complicated function $h(c)$ one could produce arbitrary structure constants $\mathcal{A}$ and $\mathcal{B}$. However, we believe that there are additional strong constraints on the possible form of $h(c)$, without being able to give an answer to that problem. If we would know the general form of $h$, we could deduce the structure constant of any algebra of type $\mathrm{W}(2,3,4, \ldots)$, satisfying eq. (3.2).

For the $\mathrm{WA}_{n-1}$-algebras all the dimensions of the null-fields are known [12] and especially the dimensions of the lowest level null-fields satisfy the quadratic equation

$$
4 n^{2} h^{2}+2 h[c-(n-1)(2 n+1)]+c(n-1)=0
$$

When we denote by $h_{1,2}$ the two solutions of this equation, then $\mathcal{A}\left(h_{1}\right)=\mathcal{A}\left(h_{2}\right)$ (as well as $\left.\mathcal{B}\left(h_{1}\right)=\mathcal{B}\left(h_{2}\right)\right)$ give in a much easier way the structure constants for the $\mathrm{WA}_{n-1}$-algebra, as 
obtained in [13] using the free-field realization of ref. [12]. In addition to $h_{1,2}$ there is a third value of $h$ that reproduces the same structure constants and that is given by

$$
h_{3}=\frac{n+1}{2} \frac{c}{c+1-n} .
$$

But we know that the $\mathrm{WA}_{n-1}$-algebra is not the only possibility for $\mathrm{W}$-algebras of this type. As has been shown in ref. [14], for the algebra $\mathrm{W}(2,3,4,5)$ exist two different solutions: One is given by $\mathrm{WA}_{4}$ and therefore described by eqs. (3.9) and (3.10). But also for the second algebra the structure constants are of the form (3.6) and (3.8) and in this case the dimensions of the highest weights satisfying eq. (3.2) are

$$
h_{1}=-\frac{1}{2} \frac{c+4}{c+1} ; \quad h_{2}=\frac{1}{8} \frac{c(2-c)}{c+1} ; \quad h_{3}=\frac{3}{2} \frac{c}{c+1} .
$$

It would therefore be interesting to find other examples for algebras of type $\mathbf{W}(2,3,4, \ldots)$ beyond $\mathrm{WA}_{n-1}$ to get a hint on the general structure of $h$. But even with our limited knowledge we shall find important consequences in the following examples.

\section{W-algebras of type $\mathrm{W}(2,4, \ldots)$}

We shall now consider algebras, where the lowest-dimensional bosonic primary field has spin 4 , hence obeying the OPE

$$
W_{4} \star W_{4}=\frac{c}{4} I+\sqrt{\mathcal{C}} W_{4}+W_{6}
$$

and that there is a highest weight $P_{o}$ with the property that

$$
\begin{aligned}
& W_{4} \star P_{o}=w_{4} P_{o} \\
& W_{6} \star P_{o}=w_{6} P_{o}+\ldots .
\end{aligned}
$$

All information we are looking for is already contained in the Jacobi-identity $\left(W_{4}, W_{4}, P_{o}\right)$; the eigenvalues $w_{4}$ and $w_{6}$ are

$$
w_{4}=-\frac{3 h\left[c^{2}\left(2 h^{2}-2 h-9\right)+3 c\left(12 h^{3}-49 h^{2}+40 h-2\right)+2 h\left(12 h^{2}+5 h-14\right)\right]}{(22+5 c) \sqrt{\mathcal{C}}[c(2 h-3)(h-2)+h(4 h-5)]}
$$




$$
w_{6}=\frac{2(c-1)(22+5 c) h[c(h+2)+(5 h-2)(3 h-4)][c(2 h+3)+4 h(12 h-7)]}{3(24+c)(2 c-1)(68+7 c)[c(2 h-3)(h-2)+h(4 h-5)]}
$$

and the structure constant $\mathcal{C}$ is given by

$\mathcal{C}(h)=\frac{36\left[c^{2}\left(2 h^{2}-2 h-9\right)+3 c\left(12 h^{3}-49 h^{2}+40 h-2\right)+2 h\left(12 h^{2}+5 h-14\right)\right]^{2}}{(22+5 c)[c(h+1)+(3 h-1)(h-2)][c(2 h+1)+2 h(8 h-5)][c(2 h-3)(h-2)+h(4 h-5)]}$

One observation we make is the fact that $\mathcal{C}=50 / 3$ for $c=1$, independent of $h$ and hence independent of the actual algebra as long as there is no pole at this value of the central charge.

We expect at least four different types of algebras of the form $\mathrm{W}(2,4, \ldots)$, that are $\mathrm{WD}_{n}$, $\mathrm{WB}(0, n), \mathrm{WB}_{n}$ and $\mathrm{WC}_{n}$ t. For both the algebras $\mathrm{WD}_{n}$ (integer $n \geq 3$ ) and $\mathrm{WB}(0, m)$ with $n=(2 m+1) / 2$ (half-integer $n \geq 5 / 2)$ we have for the null-fields the condition [15, 16]

$$
4 n(n-1) h_{1,2}^{2}+h_{1,2}(c+n(3-4 n))+(n-1) c=0
$$

equivalent to $\quad h_{3}=n$.

In addition, for $n=3 / 2$ we find the bosonic projection of the super-Virasoro algebra [6, 07].

We obtain therefore the structure constant $\mathcal{C}$ for $\mathrm{WD}_{n}$ and $\mathrm{WB}(0, n)$ by simply setting $h=n$ or $h=(2 n+1) / 2$ into eq. (4.4). We can compare the limit $c \rightarrow \infty$ of the structure constant $\mathcal{C}$ of $\mathrm{WD}_{n}$ with its known classical limit, given in ref. [17], and find agreement.

For the non-simply laced algebras $\mathrm{WB}_{n}$ and $\mathrm{WC}_{n}$ the two solutions $h_{ \pm}$of a quadratic equation no longer describe the same algebra but interchange WB and WC [18]. The dimensions $h_{1}$ and $h_{2}$ are now solutions of two different equations,

$$
\begin{aligned}
8 n(1+n) h_{1}^{2}+2 h_{1}(c-n(3+2 n))+c(2 n-1) & =0 \\
(2 n-1)(2 n+1) h_{2}^{2}+h_{2}(c-n(6 n+1))+c n+2 n^{2} & =0
\end{aligned}
$$

and there is a third equation, corresponding to $h_{3}$

$$
2(c+2 n) h_{3}^{2}-h_{3}(n(2 n+3)+c(4 n+3))+c(n+1)(2 n+1)=0,
$$

\footnotetext{
${ }^{4}$ Strictly speaking we should not include $W D_{3}$ and $W_{4}$ here since these algebras are not of the desired type because they have additional bosonic fields of dimension 3 and 4; it turns out, however, that they are described as well by the formulas.
} 
where $h_{1}, h_{2}$ and $h_{3}$ for the algebra WB are given by the $(-,+,+)$ solutions of eqs. (4.6), (4.7) and (4.8), respectively, and those for the algebra WC by the solutions of the opposite sign.

The structure constants for $\mathrm{WB}_{n}$ and $\mathrm{WC}_{n}$ are more complex and we shall therefore present them in the appendix A. Their classical limit for general $n$ coincide again with the result of ref. [17]. For $n=2$ and $n=3$ the result reproduces the structure constants of $\mathrm{WBC}_{2}$ [19] and $\mathrm{WB}_{3}$ and $\mathrm{WC}_{3}[5]$.

In all cases considered we can also ask for the limit $n \rightarrow \infty$. We find the result that

$$
\begin{aligned}
\mathcal{C}\left[\mathrm{WD}_{\infty}\right] & =\mathcal{C}[\mathrm{WB}(0, \infty)]=\mathcal{C}\left[\mathrm{WB}_{\infty}\right]=54 \frac{(3 c+2)^{2}}{(c+2)(5 c+22)} \\
\mathcal{C}\left[\mathrm{WC}_{\infty}\right] & =2 \frac{(19 c-34)^{2}}{(2 c-1)(5 c+22)}
\end{aligned}
$$

The even-dimensional fields of the algebra $W_{\infty}$ [20] form a subalgebra and it turns out that for this subalgebra the structure constant $\left(\mathcal{C}_{44}^{4}\right)^{2}$ is identical to eq. (4.9) after making a suitable change of basis that brings us from the primary fields used here to the fields where the $\mathrm{W}_{\infty}$-algebra linearizes. Since also the values for $w_{4}$ and $w_{6}$ for $\mathrm{W}_{\infty}$ and $\mathrm{WB}_{\infty}$ agree, we conjecture that $W B_{\infty}$ is the subalgebra of even-dimensional fields of $W_{\infty}$ (that is equivalent to $W A_{\infty}$ ). In the same way the algebra $W C_{\infty}$ is the subalgebra of the even-dimensional fields of $W_{1+\infty}$, constructed in ref. [21]. It is also interesting to note that these limits can also be obtained by inserting $h=1$ or $h=1 / 2$, respectively, into eq. (4.4) as these values are the solutions to eq. (4.7) in the $n \rightarrow \infty$ limit.

So far we have taken in all solutions $n$ to be positive. However, from our point of view there is no restriction on the sign of $n$ and we could as well imagine negative values: If the Jacobi-identities are satisfied for any positive $n$ they will also be satisfied for negative $n$, as long as there is no structure constant that diverges in this case. The only open question will then be, whether the algebra closes with a finite number of fields.

Indeed, there exists at least one example of an algebra that can be described by a negative $n$ : This is the "strange" solution of the algebra $\mathrm{W}(2,4,6)$ [5, 8]. One obtains the structure constants of this algebra by setting $h=n=-1$. Very formally we can therefore say that this algebra 
is $W D_{-1}$. Whether one can give to this formal notation a mathematical meaning has to be seen. The structure constant $\mathcal{C}$ does not diverge in this case in the limit $c \rightarrow \infty$ because $n$ is negative, but because $n$ takes one of four special values where $\mathcal{C}$ diverges in this limit (another is e.g. $n=3 / 2$, the bosonic projection of the super-Virasoro-algebra).

Minimal models for the algebra $\mathrm{WD}_{n}$ are given by

$$
c(n)=n-2 n(n-1)(2 n-1)\left(\frac{(p-q)^{2}}{p q}\right) .
$$

When we take our interpretation for the fourth solution of $\mathrm{W}(2,4,6)$ as $\mathrm{WD}_{-1}$ more seriously, then we should expect minimal models for this algebra, following eq. (4.11) for the central charge, to be a sub-set of

$$
c(-1)=-1+12\left(\frac{(p-q)^{2}}{p q}\right) .
$$

Indeed, all examples for minimal models found in [8] obey this form with $q=p+1$.

Even more intrigueing, we can also give a formula for the dimension $h$ in this case that is obtained by taking $n=-1$ for the highest weights of $\mathrm{WD}_{n}$ : Let

$$
x\left(\vec{l}_{i}\right)=l_{i}(p+1)-l_{i}^{\prime} p \quad \text { with } \quad \vec{l}_{i}=\left(l_{i}, l_{i}^{\prime}\right), \quad i=1,2
$$

then the dimensions of the highest weights can be written as

$$
h\left(\vec{l}_{1}, \vec{l}_{2} ; r, r^{\prime}\right)=\frac{r\left(x\left(\vec{l}_{1}\right)-1\right)\left(x\left(\vec{l}_{1}\right)+2 x\left(\vec{l}_{2}\right)-6-r\right)+r^{\prime}\left(x\left(\vec{l}_{2}\right)-1\right)\left(x\left(\vec{l}_{2}\right)-4-r^{\prime}\right)}{2 p(p+1)}
$$

where $p=2 m+1$ with positive integer $m$ and

$$
\begin{aligned}
& l_{2}=l_{1}=(1,1) \quad r=r^{\prime}=1 \\
& \text { or } \quad l_{2}=(1,1) \quad l_{1}=(1,2),(2,1),(2,2) \quad 1 \leq r \leq m-1, \quad r^{\prime}=1 \\
& \text { or } \quad l_{2}=(1,2) \quad l_{1}=(2,1) \\
& l_{2}=(2,1) \quad l_{1}=(1,2) \quad 1 \leq r<r^{\prime} \leq m-1
\end{aligned}
$$

The $m^{2}$ dimensions (4.14) reproduce the values for the examples given in ref. 22] for $m=3$ and in ref. [8] for $m=4$ and $m=5$. For $m=2$ we get the highest weights of the Virasoro minimal model at $c=-3 / 5$. However, the restrictions used here $(q=p+1, p$ odd, bounds for 
$\vec{l}_{i}$ and $r, r^{\prime}$ ) were found completely by comparing with these examples f; until we have a better understanding what we mean by "WD_-, these bounds remain mysterious.

We presented here this formal identification only for the structure constant $\mathcal{C}_{44}^{4}$, but indeed we inluded also the spin-6 field and computed all structure constants up to this field (see appendix B) and they agree with this identification (of course this is also true for the other $\mathrm{W}(2,4,6)$-algebras $)$.

It might also be interesting to note that with the ansatz that $h_{1,2}$ are given by both the solutions $h_{ \pm}$of a general quadratic equation of the form

$$
h^{2}+h\left(a_{1} c+a_{2}\right)+\left(a_{3} c+a_{4}\right)=0
$$

with free parameters $a_{1}, \ldots, a_{4}$, the algebra $\mathrm{W}(2,3,4, \ldots)$ has only one solution of this type, given by $\mathrm{WA}_{n-1}$, eq. (3.9), whereas for $\mathrm{W}(2,4, \ldots)$ we find apart from the solution of $\mathrm{WD}_{n}$ and $\mathrm{WB}(0, m)$, eq. (4.5), and the special case $\mathrm{WB}_{2}=\mathrm{WC}_{2}$ another possibility, given by

$$
\begin{aligned}
& 4 n(n-1) h_{1,2}^{2}+h_{1,2}(c+(n-1)(1-4 n))+(n-1) c+(n-1)^{2}=0 \\
& h_{3}=n \frac{c}{c+n-1} .
\end{aligned}
$$

We do not know of any example of an W-algebra connected to this case and is has to be seen, whether such an algebra exists (for some or any value of $n$ ).

\section{$5 \mathrm{~W}$-algebras of type $\mathrm{W}(2,5, \ldots)$ and type $\mathrm{W}(2,6, \ldots)$}

For the algebras of type $\mathrm{W}(2,5, \ldots)$ and $\mathrm{W}(2,6, \ldots)$ the Jacobi-identities become strong enough to determine also the dimension of the heighest weights. Among the solutions for $\mathrm{W}(2,5, \ldots)$ we would expect the algebra $\mathrm{WE}_{6}=\mathrm{W}(2,5,6,8,9,12)$. For the type $\mathrm{W}(2,6, \ldots)$ we should find the orbifold of $W_{3}$ (because of the parity-conservation of $W_{3}$, the spin- 6 composite field

\footnotetext{
${ }^{5}$ In a "normal" WD minimal model the rôles of $l, l^{\prime}$ and $r, r^{\prime}$ are interchanged: There, $r$ and $r^{\prime}$ run over a fixed range, whereas the bounds for $l$ and $l^{\prime}$ depend on $p$.
} 
$W_{6} \sim: W_{3} W_{3}:+\ldots$ spans an algebra of the desired type and for the same reason $W_{3}$ plays the rôle of $\left.P_{o}\right), \mathrm{WG}_{2}=\mathrm{W}(2,6), \mathrm{WF}_{4}=\mathrm{W}(2,6,8,12)$ and $\mathrm{WE}_{7}=\mathrm{W}(2,6,8,10,12,14,18)$.

In the case of $\mathrm{W}(2,5, \ldots)$ in the simplest Jacobi-identity $\left(W_{5}, W_{5}, P_{o}\right)$ does not enter a structure constant and we find a $5^{\text {th }}$-order condition for $h(c)$. For W $(2,6, \ldots)$ we find the structure constant $\mathcal{C}_{66}^{6}(h)$ and the solutions for $h$

$$
\begin{aligned}
h=3 & \text { (for the orbifold of } \left.\mathbf{W}_{3}\right) ; \\
28 h^{2}+h(c-26)+3(c+2)=0 & \text { leading to the structure constant of } \mathbf{W G}_{2}, \\
& \text { as given in [23, 24, 5]; }
\end{aligned}
$$

as well as a third order equation,

$$
88+20 c-64 h-13 c h-22 h^{2}+2 c h^{2}+16 h^{3}=0
$$

and a $6^{\text {th }}$-order condition.

However, it is doubtful that the WE-algebras are contained in these solutions for $\mathrm{W}(2,5, \ldots)$ and $\mathrm{W}(2,6, \ldots)$, since the algebras $\mathrm{WE}_{i}$ are simply laced we would therefore expect that $h$ again satisfies a quadratic equation. Also the algebra $\mathrm{WF}_{4}$ is missing: There is no spin-10 field in $\mathrm{WF}_{4}$, but only our solution corresponding to $W G_{2}$ is without it.

We have also to remember that we are only looking at one Jacobi-identity $\left(\left(W_{5}, W_{5}, P_{o}\right)\right.$ or $\left(W_{6}, W_{6}, P_{o}\right)$, respectively) at this point and we therefore get necessary conditions for $h$. It is not at all evident that the solutions to the $5^{\text {th }}$-order condition in the case of $\mathrm{W}(2,5, \ldots)$ and the $3^{\text {rd }}$-order and the $6^{\text {th }}$-order condition for $\mathrm{W}(2,6, \ldots)$ lead to $\mathrm{W}$-algebras.

One reason why $\mathrm{WF}_{4}\left(\right.$ and $\mathrm{WE}_{i}$ ?) are missing might be that the condition that all the fields $P_{i}$, $i \neq 0$, drop out of the OPE of the $W$-fields with $P_{o}$ simultaneously is no longer satisfied. 


\section{Conclusions}

All calculations are based on the assumption that there exists a highest weight $P_{o}$ such that its $\mathrm{OPE}$ with the lowest-dimensional bosonic generator $W$ obeys the simple rule $W \star P_{o}=$ $w P_{o}$. When we consider as an example the $\mathrm{WA}_{n-1}$-algebra, the fields with even parity form a subalgebra of the type $\mathrm{W}(2,4, \ldots)$. However, as it is clear from eq. (3.2), the field $P_{3}$ given by eq. (3.3) is not of the form we need for null-states and therefore a reduction to eq. (4.2) will only happen accidentally. By taking the solution e.g. for $\mathrm{WA}_{n-1},(n \geq 3$ integer $)$, this condition is only satisfied for $n=4$ and $n=\infty$. We see that our simple ansatz will not be be true in general for orbifold constructions except single cases (see examples given).

Moreover, instead of our original ansatz we could think of a different one of the form $W \star P_{o}=$ $w P_{1}$ (or other fields than $P_{o}$ ). Such an ansatz would be automatically needed for example if $W$ is fermionic. We have not studied whether in the cases considered here a different ansatz would lead to different solutions. In some examples we checked that it reproduced known results (with different values of $h$ ). However, it seems likely that for the algebras WE and WF we need a even more complicated ansatz.

Another draw-back of our approach is that we are not able to deduce the field content of our algebras. This has to be obtained by other means. On the other hand, we were able to obtain structure constants for a variety of algebras, like $\mathrm{WD}_{n}$ or $\mathrm{WB}_{n}$ as functions of $n$, a task that would be principally impossible by evaluating the Jacobi-identities of the algebra itself.

Moreover it is no longer necessary to give to each single algebra a new set of solution of the structure constants, but it is sufficient to write down the general function $\mathcal{C}(h)$ and then for each algebra once its form of $h(c)$ (this is especially true for W-algebras of the same type).

The calculations up to the point presented are relatively simple. We could also get higher structure constants in the same way, but then calculations will turn out to be more cumbersome because then also composite primary fields of the algebra will contribute (that did not enter so far). 
Of course also other examples can be considered, for example algebras with a set of several spin-4 fields etc. It would also be interesting to find other examples that correspond to negative $n$ in eq. (4.5).

\section{Acknowledgements}

Calculations have been carried out with the Mathematica-package for calculating operator product expansions by K. Thielemans [25].

I would like to thank W. Eholzer, A. Honecker, R. Hübel and E. Ragoucy for helpful discussions and comments on the manuscript.

I am grateful to the University of Torino for kind hospitality during this work and to the Laboratoire de Physique Théorique ENSLAPP, Groupe d'Annecy, during its final stage. 


\section{A Structure constant for $\mathrm{WB}_{n}$ and $\mathrm{WC}_{n}$}

The structure constant $\mathcal{C}$ (see eq. (4.1)) for $\mathrm{WB}_{n}$ and $\mathrm{WC}_{n}$ has the general form

$$
\mathcal{C}\left[\mathrm{WB}_{n}, \mathrm{WC}_{n}\right]=\frac{g_{1} \pm g_{2} \sqrt{y}}{g_{3}}
$$

where

$$
\begin{aligned}
g_{1}= & c^{6}\left(-1694+1191 n+2202 n^{2}-712 n^{3}-672 n^{4}+96 n^{5}+64 n^{6}\right)+ \\
& 3 c^{5}\left(110-12167 n-9349 n^{2}+20942 n^{3}+4816 n^{4}-8920 n^{5}-432 n^{6}+960 n^{7}\right)+ \\
& 3 c^{4}\left(1024+10626 n-68841 n^{2}-193562 n^{3}+32218 n^{4}+163136 n^{5}-58656 n^{6}\right. \\
& \left.\quad-32672 n^{7}+12704 n^{8}\right)+ \\
& 2 c^{3}\left(-2204+8274 n+225492 n^{2}+384408 n^{3}-866403 n^{4}-1414656 n^{5}+469512 n^{6}\right. \\
& \left.\quad+436560 n^{7}-340080 n^{8}+54208 n^{9}\right)+ \\
& 12 c^{2} n\left(-3118-21773 n+8646 n^{2}+314624 n^{3}+318922 n^{4}-377096 n^{5}\right. \\
& \left.\quad-345264 n^{6}+77088 n^{7}-1760 n^{8}\right)+ \\
& 24 c n^{2}\left(-1260-13824 n-73493 n^{2}+3500 n^{3}+269320 n^{4}+199120 n^{5}-14800 n^{6}-8192 n^{7}\right)+ \\
& 32 n^{3}\left(-1960-6090 n-39153 n^{2}-88436 n^{3}-56328 n^{4}+2256 n^{5}+8816 n^{6}\right) \\
g_{2}= & (n-2)(1-c)(22+5 c)^{2}\left(5 c+28 n+2 c n+40 n^{2}\right)\left(c+4 c n+6 n^{2}+8 n^{3}+8 n^{4}\right) \\
g_{3}= & (n-1)(-1+2 c)(22+5 c)\left(2 c-5 n+c n+2 n^{2}\right)\left(2+c+11 n+c n+12 n^{2}\right) \\
& \left(3 c+10 n+2 c n+12 n^{2}\right)\left(3 c+20 n-8 c n+8 n^{2}+4 c n^{2}\right)
\end{aligned}
$$

and

$$
y=c^{2}+2 c n\left(1-6 n-8 n^{2}\right)+n^{2}(3+2 n)^{2} .
$$

For the algebra $\mathrm{WB}_{n}$ we have to take the "-", for the algebra $\mathrm{WC}_{n}$ the "+"-sign in (A.1). 


\section{B Structure constants for $\mathrm{W}(2,4,6, \ldots)$}

For the algebra with OPEs

$$
\begin{aligned}
& W_{4} \star W_{4}=\frac{c}{4} I+\sqrt{\mathcal{C}} W_{4}+\sqrt{\mathcal{D}} W_{6} \\
& W_{4} \star W_{6}=\frac{2}{3} \sqrt{\mathcal{D}} W_{4}+\mathcal{E} \sqrt{\mathcal{C}} W_{6}+W_{8,1} \\
& W_{6} \star W_{6}=\frac{c}{6} I+\frac{2}{3} \mathcal{E} \sqrt{\mathcal{C}} W_{4}+\mathcal{F} \sqrt{\mathcal{D}} W_{6}+W_{8,2}+W_{10}
\end{aligned}
$$

we find in addition to the structure constant $\mathcal{C}$, eq. (4.4), the structure constants

$$
\begin{aligned}
& \mathcal{D}(h)=12 \frac{(c-1)(22+5 c)^{2}[c(h+2)+(5 h-2)(3 h-4)]}{(24+c)(2 c-1)(68+7 c)[c(h+1)+(3 h-1)(h-2)]} \times \\
& \frac{[c(2 h+3)+4 h(12 h-7)][c(2 h-5)(h-3)+2 h(4 h-7)]}{[c(2 h+1)+2 h(8 h-5)][c(2 h-3)(h-2)+h(4 h-5)]} \\
& \mathcal{E}(h)= \\
& \frac{(2 c-1)(68+7 c)(h-4)[c(h+3)+4 h(12 h-13)]}{6(24+c)\left[c^{2}\left(2 h^{2}-2 h-9\right)+3 c\left(12 h^{3}-49 h^{2}+40 h-2\right)+2 h\left(12 h^{2}+5 h-14\right)\right]} \\
& \mathcal{F}(h)=\frac{5}{9} \frac{1}{(c-1)(24+c)(22+5 c)} \times \\
& \frac{1}{[c(h+2)+(5 h-2)(3 h-4)][c(2 h+3)+4 h(12 h-7)][c(2 h-5)(h-3)+2 h(4 h-7)]} \times \\
& \left\{4 c^{6}\left(-9-h+h^{2}\right)\left(-87-4 h+4 h^{2}\right)+\right. \\
& 3 c^{5}\left(17016-32683 h+36363 h^{2}-2692 h^{3}-3732 h^{4}+656 h^{5}\right)+ \\
& 3 c^{4}\left(10488-537784 h+912383 h^{2}-779183 h^{3}+510680 h^{4}-155788 h^{5}+16704 h^{6}\right)+ \\
& 24 c^{3}\left(-3900+39820 h+567630 h^{2}-1411325 h^{3}+1089288 h^{4}-319940 h^{5}+34032 h^{6}\right)+ \\
& 24 c^{2}\left(-41856+100768 h-554146 h^{2}+1010616 h^{3}-696919 h^{4}+127816 h^{5}+20976 h^{6}\right)+ \\
& 96 c h\left(113632-496572 h+799600 h^{2}-505275 h^{3}+124168 h^{4}-15600 h^{5}\right)+ \\
& \left.1024 h^{2}\left(-10759+53477 h-94159 h^{2}+66156 h^{3}-15696 h^{4}\right)\right\} .
\end{aligned}
$$

For the algebra $\mathrm{W}(2,4,6)$ this is the complete set of structure constants among the simple fields. Especially they agree with the ones given in [5] for the bosonic projection of the super-Virasoro algebra for $h=n=3 / 2$ and with the special fourth solution for $h=n=-1$. 


\section{References}

[1] L. Fehér, L. O'Raifeartaigh, P. Ruelle, I. Tsutsui and A. Wipf, Phys. Rep. 222 (1992) 1

[2] L. Frappat, E. Ragoucy and P. Sorba, "W-algebras and superalgebras from constrained WZW models: A group theoretical classification", ENSLAPP-AL-391-92, to appear in Communications in Mathematical Physics

[3] P. Bowcock and G.M.T. Watts, Nucl. Phys. B379 (1992) 63

[4] P. Bouwknegt and K. Schoutens, Phys. Rep. 223 (1993) 183

[5] H.G. Kausch and G.M.T. Watts, Nucl. Phys. B354 (1991) 740

[6] P. Bouwknegt, "Extended conformal algebras from Kac-Moody algebras" in "Infinitedimensional Lie algebras and Lie groups", ed. V. Kac, Proc. CIRM-Luminy conference 1988, World Scientific Singapore (1989)

[7] H.G. Kausch, "Chiral Algebras in Conformal Field Theory", PhD-Thesis at the University of Cambridge (1991)

[8] W. Eholzer, A. Honecker and R. Hübel, Phys. Lett. 308B (1993) 42

[9] A.A. Belavin, A.M. Polyakov and A.B. Zamolodchikov, Nucl. Phys. B241 (1984) 333

[10] A.B. Zamolodchikov, Teor. Mat. Fiz. 65 (1985) 1205

[11] V.A. Fateev and A.B. Zamolodchikov, Nucl. Phys. B280 [FS18] (1987) 644

[12] V.A. Fateev and S.L. Lukyanov, Int. J. Mod. Phys. A3 (1988) 507

[13] K. Hornfeck, Phys. Lett. 275B (1992) 355

[14] K. Hornfeck, "W-algebras with set of primary fields of dimensions $(3,4,5)$ and $(3,4,5,6)$ ", KCL-TH-92-9, DFFT-70/92, hep-th/9212104, to appear in Nuclear Physics B

[15] V.A. Fateev and S.L. Lukyanov, Sov. J. Nucl. Phys. 49 (1989) 925

[16] V.A. Fateev and S.L. Lukyanov, Sov. Sci. Rev. A Phys. 15 (1990) 1 
[17] L. Frappat, E. Ragoucy and P. Sorba, "Folding the W-algebras", ENSLAPP-AL-408/92, to appear in Nuclear Physics B

[18] E. Frenkel, V. Kac and M. Wakimoto, Comm. Math. Phys. 147 (1992) 295

[19] P. Bouwknegt, Phys. Lett. 207B (1988) 295

[20] C.N. Pope, L.J. Romans and X. Shen, Nucl. Phys. B339 (1990) 191

[21] C.N. Pope, L.J. Romans and X. Shen, Phys. Lett. 242B (1990) 401

[22] W. Eholzer, M. Flohr, A. Honecker, R. Hübel, W. Nahm and R. Varnhagen, Nucl. Phys. B383 (1992) 249

[23] J.M. Figueroa-O'Farrill and S. Schrans, Phys. Lett. 245B (1991) 471

[24] R. Blumenhagen, M. Flohr, A. Kliem, W. Nahm, A. Recknagel and R. Varnhagen, Nucl. Phys. B361 (1991) 255

[25] K. Thielemans, Int. J. Mod. Phys. C2 (1991) 787 